\title{
Improving non-invasive ventilation documentation
}

\author{
Matthew Smith, Natalie Elkheir
}

St Georges Hospital

\begin{abstract}
Record keeping for patients on non-invasive ventilation (NIV) at St. Georges Hospital is poor. The initial NIV prescription is often not recorded, and changes to the NIV prescription or the rationale for the changes (ABG results) are also poorly documented. This leads to confusion for nurses/doctors as to what the correct settings are, meaning patients could receive ineffective ventilation. The use of NIV is also poorly recorded by nursing staff meaning that doctors are unsure if the prescribed NIV is being achieved. This can lead to treatment being escalated unnecessarily in the event of treatment failure.
\end{abstract}

Non-invasive ventilation (NIV) is the provision of ventilatory support in the form of positive pressure via the patient's upper airway using a mask or similar device. NIV is indicated for treatment of acute hypercapnic respiratory failure, of which there are many causes, though COPD is the indication in up to $70 \%$ of cases.[1] British Thoracic Society (BTS) guidelines for NIV suggest that the rationale for commencing a patient on NIV and the proposed settings should be clearly documented.[2] Clinicians cannot effectively tailor changes to the patients NIV settings if this information is not clearly recorded, which could lead to increased time requiring NIV or NIV failure.

Three main areas were considered important to measure for this project. The initial prescription of the NIV, changes to the NIV settings, and nursing documentation surrounding NIV. A baseline measurement of NIV documentation for two weeks found NIV documentation to globally very poor. NIV was formally prescribed $29 \%$ of the time, full detail of intended settings were documented $57 \%$ of the time, the decision to commence NIV was discussed with the respiratory consultant/SpR just $29 \%$ of the time and on no occasion was a decision regarding escalation of treatment recorded. Eighteen changes were made to the NIV settings. These were formally prescribed $22 \%$ of the time and detail of the intended settings was recorded $44 \%$ of the time. Nursing documentation included detail on the length of NIV use just $21 \%$ of the time, and comments on the NIV use were left just $33 \%$ of the time.

The intervention was a unified four page NIV prescription chart. Page 1: An area for the NIV to be initially prescribed with reminder questions for important considerations. Page 2: An area for changes to be made to the NIV settings. Page 3: An area for the most up to date NIV/respiratory plan to be documented. Page 4: Nursing documentation, with prompts for the time NIV was put on/off and for comments. The chart was printed at St Georges print services and paid for by the respiratory ward.

A further two weeks of monitoring followed after implementation of the new chart. Improvements were seen in all areas of documentation surrounding NIV. The NIV was formally prescribed $86 \%$ of the time compared to $29 \%$ pre-intervention. The NIV settings were stated $100 \%$ of the time in the initial prescription. An escalation decision was recorded $71 \%$ of the time. Changes to the NIV settings improved to $92 \%$. Nursing documentation improved greatly. The length of the NIV use was recorded for $91 \%$ of NIV days compared to $21 \%$ previously ( $p<0.001$ ), and comments on the use of NIV improved from from $33 \%$ to $98 \%(p<0.001)$. Cases where the documentation remained poor were in those patients for whom the new chart had not been used.

The implementation of a unified NIV prescription chart to be kept in the bedside notes on the respiratory ward greatly improved documentation surrounding NIV. Further work must be done to ensure that the chart is used $100 \%$ of the time. This will include adjusting the chart to reduce the workload to use it, rolling the chart out on the acute medical ward, and submitting the chart to the trust board for its acceptance as an official trust chart.

\section{Problem}

Record keeping for patients on non-invasive ventilation (NIV) at St. Georges Hospital is often very poor. The initial NIV prescription is often not recorded, nor are subsequent changes to the NIV prescription/settings or the rationale for the changes, such as arterial blood gas (ABG) results. This can lead to confusion for both the nursing staff and medial staff as to what the correct settings are, meaning the patient could receive ineffective ventilation. Whether the intended prescribed NIV use is met is also often poorly recorded by nursing staff. This means that medical staff are unaware whether the prescribed amount of NIV is actually being achieved. This is a particular problem overnight, as the night nursing staff are not present during the day to ask. This can lead to treatment being escalated unnecessarily.

To assess the scope of the problem prior to the start of the project, a snapshot audit was taken of the NIV documentation on the respiratory ward at St. Georges Hospital. All patients on NIV at the time of audit were included, and documentation on NIV from their 
BMJ Quality Improvement Reports

entire time on the treatment was reviewed. There were seven patients on NIV on the respiratory ward at the time of audit. The Initial $A B G$ was recorded in all seven. The respiratory team (consultant or SpR) was involved in four out of seven $(57.1 \%$ ) instances. NIV was formally prescribed in two out of seven $(28.6 \%)$ instances. Of these, IPAP, EPAP and FiO2 were recorded fully, although intended use was only recorded in one of seven $(14.3 \%)$ instances.

A decision was made regarding escalation of NIV in the event of treatment failure was made in four patients (57.1\%). There had been 21 changes to NIV settings in total. Of these, the repeat ABG guiding the changes to settings was recorded in 14 (66.6\%) occasions, and the decision to change the settings was recorded in $11(52.4 \%)$. Changes were not made to the formal NIV prescription in any of the 21 instances. There were a total of 102 days of NIV use. Nurse documentation made reference to the use of NIV on $68 / 102(66.6 \%)$ days. More specific information such as time the NIV was started (14.7\%), time NIV was stopped $(9.8 \%)$ or the length of NIV use if time on/off was not given (8.8\%) was more sporadically documented.

\section{Background}

Non-invasive ventilation (NIV) is the provision of ventilatory support in the form of positive pressure via the patient's upper airway using a mask or similar device. It has a number of advantages over invasive ventilation techniques (eg tracheal intubation or the use of laryngeal mask airways) such as a reduced morbidity and mortality. It can be provided in critical care areas other than intensive care, eg a respiratory ward, and at an earlier stage. This nature of NIV also means that it can be used on an intermittent basis. NIV is indicated for treatment of acute hypercapnic respiratory failure, COPD with a respiratory acidosis, hypercapnic respiratory failure secondary to chest wall deformity or neuromuscular disease, cardiogenic pulmonary oedema unresponsive to continuous positive airway pressure (CPAP), obesity hypoventilation syndrome and weaning from tracheal intubation.[2] COPD is the indication in up to $70 \%$ of cases.[1]

British Thoracic Society (BTS) guidelines for NIV suggest that knowledge of a baseline arterial blood gas (ABG) is critical to the application of NIV, and a repeat sample should usually be taken after a short interval ( 1 to 2 and 4 to 6 hours) to see if NIV is still indicated and that these results should be recorded.[2] Guidelines also state that assessment of patient state while on NIV cannot be made in the absence of information regarding the inspired oxygen concentration, and that it is vital that the metered flow rate and the mode of supplementation (eg NIV settings and length of use) are clearly recorded.[2] If this information is not clearly recorded then clinicians cannot effectively tailor changes to the patients NIV settings, which could lead to increased time requiring NIV or NIV failure. Guidelines also state that a decision regarding escalation of treatment in the event of failure must be made, however a recent BTS audit found that the medical notes documented a plan for NIV failure just $76 \%$ of the time.[1]

\section{Baseline measurement}

Three main areas were considered important to measure for this project. The first revolves around the initial prescription of the NIV: was the initial ABG result and the decision to esclate to NIV documented, were the initial prescribed NIV settings including $\mathrm{FiO} 2$ and intended use documented, and crucially was a decision regarding escalation in the event of treatment failure documented? The second important area of measurement is whether repeat $A B G$ results were recorded, and were any subsequent changes to the NIV settings prescribed/documented? The final area of measurement is whether the nursing staff had documented the use of NIV, including any explanation as to why the intended NIV use was not achieved.

All patients on NIV on the respiratory ward were included in the baseline measurement. Data was collected daily for two weeks. NIV was formally prescribed $29 \%$ of the time. Full detail of intended settings, including IPAP, EPAP, and FiO2, were documented 57\% of the time, while the decision to commence NIV was discussed with the respiratory consultant/SpR just $29 \%$ of the time and on no occasion was a decision regarding escalation of treatment recorded. Eighteen changes were made to the NIV settings. These were formally prescribed $22 \%$ of the time and detail of the intended settings was recorded $44 \%$ of the time. Nursing documentation included detail on the length of NIV use just $21 \%$ of the time, and comments on the NIV use were left just $33 \%$ of the time.

See supplementary file: ds3727.pptx - "Pre-Intervention Results"

\section{Design}

The intervention will be a unified NIV prescription chart. Page 1 of the chart will focus on the detail of the initial prescription of NIV. There will be sections to record the arterial blood gas result prior to initiation of NIV and a section to record the initial prescribed NIV settings. There will also be a prescription checklist, with questions to act as reminders of important considerations for the doctor initiating the NIV: is there a respiratory acidosis despite maximal medical therapy? Has the patient been discussed with the respiratory consultant /SpR on call? Has a decision surrounding escalation in the event of treatment failure been made? Has the patient been discussed with the ICU consultant/SpR on call? Page 2 will provide an area for doctors to record changes to the NIV prescription, and also to record the corresponding arterial blood gas result that has prompted the changes. Page 3 of the chart will provide an area for the respiratory team to record the up to date NIV plan. The final page of the chart will be for nursing documentation of the use of the NIV; particularly the time the NIV was put on and taken off, and and additional comments, eg how well the NIV was tolerated.

The chart will be developed and then printed by the St. Georges University print services. The cost of the printing will be paid for from the respiratory ward budget. Once printed, the chart will be rolled out onto the respiratory ward. The chart will be kept in the existing bedside folder, with other documents such as observation 
BMJ Quality Improvement Reports

charts. This will ensure ease of use by the nursing staff, and ease of access by the prescribing doctor; as they will be at the patients bedside when making changes to NIV settings. Prior to implementation of the chart the doctors and nurses working on the respiratory ward will be educated about the use of the chart. To ensure sustainability of the chart the respiratory specialist nurse will act as "champion" of the chart and educate new staff members on its use as they arrive.

\section{Strategy}

The main stakeholders are the respiratory consultants, the ward matron, the respiratory specialist nurse, and the doctors and nurses working on the respiratory ward. The consultants were engaged by involving them in the development process of the chart. The ward matron and respiratory specialist nurse were already engaged in the implementation of a new chart, as they both recognised the need for an improved NIV documentation. Their continued engagement was maintained by involving them at each stage of the project. The ward staff (doctors and nurses) were engaged by highlighting the benefits of the new chart, education on its use, and an opportunity to be involved in its development and implementation, with opportunities to suggest changes/improvements. The chart underwent several revisions during its development. After each version of the chart was produced, it was presented to the main stakeholders for suggested improvements.

See supplementary file: ds3729.pdf - "Final NIV prescription sheet"

\section{Post-measurement}

After the initial two weeks gathering baseline data, the new NIV chart was implemented. A further two weeks of monitoring of NIV documentation followed to assess the effectiveness of the new chart. A nurse teaching day was attended prior to implementation to educate the nursing staff on how to use the chart, and the ward doctors were introduced to the chart and informed of its introduction to the ward. The data collection was performed by two Foundation Year 1 doctors that were not members of the respiratory team or working on the respiratory ward.

Improvements were seen in all areas of documentation surrounding NIV. The NIV was formally prescribed $86 \%$ of the time compared to $29 \%$ pre-intervention. The NIV settings (IPAP, EPAP and $\mathrm{FiO} 2$ ) were stated $100 \%$ of the time in the initial prescription vs. $71 \%$, $71 \%$, and $57 \%$ pre-intervention. Significant improvement was also seen in the documentation of a decision regarding escalation of treatment $(71 \%$ Vs. $0 \%, p=0.02)$. There were 12 changes to the NIV settings during the study period. These were formally prescribed $50 \%$ of the time compared to $22 \%$ prior to the use of the NIV chart. Of these changes, IPAP and EPAP was recorded $92 \%$ of the time compared to $50 \%$ previously $(p<0.05)$. The recording of $\mathrm{FiO} 2$ also improved but to a lesser degree (67\% from $44 \%$ ). Nursing documentation improved greatly. The length of the NIV use was recorded for $91 \%$ of NIV days compared to $21 \%$ previously $(p<0.001)$, and comments on the use of NIV improved from from
$33 \%$ to $98 \%(p<0.001)$.

See supplementary file: ds3728.pptx - "Post-Intervention Results"

\section{Lessons and limitations}

Many lessons were learnt from undertaking this project. Identification of the key stakeholders, in this case the ward matron, respiratory specialist nurse, and consultant body was essential in ensuring the new chart was developed and used. Educating the nurses and junior doctors on the use of the chart and involving them in the development process was also key to the charts success. One limitation of the chart which has come to light is that recording $A B G$ results in the chart is a duplication of effort since they are also recorded in the notes, which ultimately acted as a barrier for its use. This will be taken into consideration in future versions of the chart, with a tick box option to indicate whether the ABG result has been recorded elsewhere. It was noted that situations where documentation remained poor were those cases in which the new chart was not used. One reason for this could be that only doctors working on the respiratory ward were educated on its use. This means that doctors may not have been aware of the new chart when initiating NIV during evenings and at weekends when the respiratory ward is covered by general medical on call staff. This highlighted the importance of considering all users of the chart before undertaking the project.

\section{Conclusion}

The implementation of a unified NIV prescription chart to be kept in the bedside notes on the respiratory ward greatly improved documentation surrounding NIV. Situations where documentation remained poor were those in which the new chart was not used. Therefore further work must be done to ensure that the chart is used $100 \%$ of the time. This will include adjusting the chart to reduce the workload to use it, educating all members of medical staff on its existence and use, rolling the chart out on the acute medical ward, and submitting the chart to the trust board for its acceptance as an official trust chart.

\section{References}

1. Davies M. British Thoracic Society NIV Audit 2012. https://www.brit-thoracic.org.uk/document-library/audit-andquality-improvement/audit-reports/bts-adult-niv-auditreport-2012/.

2. British Thoracic Society Standards of Care Committee. BTS guideline: Non-invasive ventilation in acute respiratory failure. Thorax 2002;57:192-211.

\section{Declaration of interests}

Nothing to declare.

\section{Acknowledgements}


BMJ Quality Improvement Reports

We would like to thank: Helen Freedman, Lisa Higgs, Dr Yee Ean

Ong, Dr Imran Qureshi, and Dr Caroline Johnston. 